\section{A Computational Model of Maxwell's Distribution for Undergraduates}

\author{
Klaudio Peqini iD \\ University of Tirana, Albania \\ klaudio.peqini@fshn.edu.al \\ Rudina Osmanaj i \\ University of Tirana, Albania \\ rudina.zeqirllari@fshn.edu.al
}

\begin{abstract}
In this paper we employ a numerical approach to perform simulations of Maxwell distribution for several dimensionalities, based on the Central Limit Theorem. We show that by increasing the number of molecules of the gas $\mathrm{N}$, the simulated distributions tend toward the respective theoretical distributions. Also, we observed that by increasing the model temperature $\mathrm{n}$, the distribution shifted toward higher speeds, in agreement with theoretical results. The numerical simulations provide a physical definition of the concept of temperature. The codes used to perform the simulations are quite easy to construct and implement, while the results strikingly satisfy theoretical expectations. Furthermore, the actual approach makes it possible to skip the mathematical details and explain the distribution by just following the algorithm of simulations. We recommend such approach as a demonstrative tool that can be shown in a lecture class thus enriching the teaching quality and improving students' understanding.
\end{abstract}

\section{Keywords}

Maxwell's distribution, random numbers, ideal gas, kinetic theory, MATLAB

- Received 20 October 2020 • Revised 31 October 2021 • Accepted 23 December 2021

\section{Introduction}

The kinetic theory for gases has proved to be a very successful framework for the ideal gas model (Huang, 1987; van Kampen, 2007; Lemons, 2013; Serway \& Jewett, 2014). This theory, which combines mechanics and statistics, represents also a pedagogical elegance that has shaped the classical view of physical phenomena (Huang, 1987; van Kampen, 2007). The focus in an introductory Thermodynamics course is the ideal gas. The assumptions of the ideal gas are quite simple. The structural particles of the gas are material points, i.e. have no volume. Another assumption is that the structural particles collide elastically with the walls of the container meaning that no energy is lost into heat or other non-mechanical forms. Finally, the structural particles do not interact, i.e. these particles (from now on molecules) possess only kinetic energy (Huang, 1987; van Kampen, 2007; Serway \& Jewett, 2014). Despite being very simple and somehow nonrealistic the ideal gas model results to be in many situations a very good approximation, especially succeeding in interpreting quantities like pressure and its relation to other physical quantities that characterize the state of gas (Lemons, 2013; Reichl, 2016).

In the present paper we investigate only the equilibrium state of the ideal gas, in which case the thermodynamic temperature is well defined. The kinetic theory typically defines the temperature $T$, as a measure of the average kinetic energy of the molecules of the gas (Lemons, 2013). However, the molecules themselves possess different amount of kinetic energy. Recall that the molecules of the ideal gas possess only kinetic energy which is related to speed. Therefore, we can state that they do not have the same speed. The distribution of molecules with respect to speed has been establishes since 1860 by Maxwell in the framework of the kinetic theory (Reichl, 2016; Schmidt-Böcking et al., 2016). The distribution was derived by Boltzmann in 1877 (Huang, 1987; Serway \& Jewett, 2014), under the framework of statistical physics and was generalized further, exposing the Maxwell's distribution as a special case of the more general MaxwellBoltzmann distribution (Reichl, 2016).

It took a long time, six decades, and an ingeniously designed experiment known as the SternZartman experiment, to provide the first experimental evidence of the Maxwell's distribution in a gas (Schmidt-Böcking et al., 2016). This delay in the experimental confirmation of this famous theoretical result emphasizes the distinct technical difficulties. In the Thermodynamics Laboratory curriculum may be present an experimental set-up that verifies the Maxwell's distribution. Instead of a gas, there is used a "gas" of small hard spheres. However, this set-up may not be available for various reasons. This technical limitation usually is reflected in the understanding of the students being merely confined to theoretical aspects.

Computational methods are an integral and essential part of modern undergraduate physics curricula. The various techniques studied at this level are then broadened and specialized in many disciplines in graduate studies (Daineko et al., 2017; Magana \& Silva-Coutinho, 2017; Behringer \& Engelhardt, 2017). The computer-based techniques are considered crucial in tackling complicated problems that lack analytical results or in simulating complex systems whose dynamics is yet not well understood (Zupančič \& Sodja, 2013; Sarabando et al., 2014; Taub et al., 2015; Daineko et al., 2017; Magana \& Silva-Coutinho, 2017; Behringer \& Engelhardt, 2017; Yetilmezsoy \& Mungan, 2018). These techniques are widely used in areas like structural mechanics, fluid mechanics, statistical mechanics, system dynamics, etc, (Zupančič \& Sodja, 2013; Behringer \& Engelhardt, 2017). However, at university level, the computational methods are intended to be used jointly with analytical techniques. The important principles of a given discipline are initially discussed theoretically and after some clarifying analytical examples, the 
real-world problems are solved by using numerical techniques. It is reported that the combination of these two approaches considerably increases the student's understanding (Zupančič \& Sodja, 2013; Sarabando et al., 2014; Taub et al., 2015; Yetilmezsoy \& Mungan, 2018; Boriçi, 2019).

Numerical techniques are very suitable for thermodynamics or statistical physics in simulating many-body systems (van Kampen, 2007; Lemons, 2013; Reichl, 2016). Such methods are not intended to replace the experiment-oriented parts of a given course, like the laboratory sessions. Rather they should be seen as an additional approach to facilitate the exposition of the material discussed in the course, thus making it easier for students to understand what is being discussed. This is extremely useful especially when the simulations can be performed with a conventional desktop or laptop. Experimental set-ups that enable simple studies related to Maxwell's distribution may be quite expensive and may be no that helpful for students' understanding of the underlying physics (Lemons, 2014; Reichl, 2016). We strongly believe that the combination with simulations is very effective in conveying the core ideas of Maxwell's distribution. In the present paper we show the effectiveness of the numerical approach in illustrating key aspects of Maxwell's distribution for an ideal gas of various dimensionalities. We show that many of the theoretical features of the distribution are reproduced successfully. The authors of the paper teach the material presented here in a course of introductory Computational Physics taught at the Faculty of Natural Sciences, University of Tirana (Boriçi, 2019).

The paper is organized as follows: in the section 2 we discuss concisely the theory behind Maxwell's distribution and derive the key formulas for the one-, two- and three-dimensional ideal gas in equilibrium at a given temperature $T$. In section 3 we discuss the algorithm of the simulations. In section 4 we report some results regarding the physical accuracy of the simulations and their effectiveness in visualizing key features of Maxwell's distribution. In section 5 we discuss some of the results reported in the previous section. In section 6 we discuss the paper from the pedagogical point of view providing some helpful suggestions. Finally we conclude the paper with the conclusions section.

\section{Maxwell's distribution for different dimensionalities}

One of the key properties of the ideal gas is its independence from frame's spatial orientation, i.e. it is isotropic. The ideal gas looks the same in whatever direction you look or despite the frame chosen by the observer (van Kampen, 2007; Serway \& Jewett, 2014). Therefore, the different velocity components possess the same properties and are characterized by the same distribution. This fact is very important and pedagogically has been traditionally used to obtain the Maxwell distribution in three dimensions. Actually this very argument can be used to obtain the Maxwell distribution in any dimensionality (Lemons, 2013; Reichl, 2016). The isotropy of the ideal gas makes it possible to neglect the direction and focus only on the magnitude of the velocity, because it is basically a measure of the energy of the molecule (Huang, 1987; van Kampen, 2007; Lemons,
2013; Serway \& Jewett, 2014; Reichl, 2016). The one-dimensional case is crucial for higher dimensionalities. Therefore, we will start with the ideal gas in one dimension and then will discuss the two- and three-dimensional gas.

\section{One-dimensional ideal gas}

The Maxwell's distribution is a special case of the more general Boltzmann distribution, obtained by the latter from statistical considerations. In both cases there is considered a physical system, like a gas, being in thermal equilibrium with temperature $T$. The Boltzmann distribution describes the probability $P_{i}$ for a given subsystem, like one molecule of the gas, to be in the state $i$, with respective energy $\varepsilon_{i}$ :

$$
P_{i}=P\left(\varepsilon_{i}\right) \propto e^{-\frac{\varepsilon_{i}}{k_{B} T}},
$$

where $\varepsilon_{i}$ is the energy of the state $i, k_{B}$ is the Boltzmann constant and $T$ is the thermodynamic temperature. In the relation (1) it is assumed that the system has a discrete number of levels. In the continuous case, we discuss the probability for a given molecule to have its energy in a given range rather than a single value. When this range is the infinitesimally small quantity $d \varepsilon$, the respective probability is the infinitesimally small amount $d P$. These two quantities are proportional to each other and the proportionality coefficient is known as the probability density function (PDF), $g(v)$ (Huang, 1987; van Kampen, 2007; Lemons, 2013; Reichl, 2016). The PDF of the Boltzmann distribution is proportional to the exponential function in (1)

The Maxwell's distribution pertains to the continuous distribution category and describes the distribution of molecules of the ideal gas for different energy values. Since these molecules possess only kinetic energy, for a one-dimensional gas there can be written

$$
\varepsilon=\frac{1}{2} m v_{x}^{2} \text {. }
$$

By substituting (2) into the continuous form of (1) and introducing a proportionality constant $C$ in (1), we have

$$
g\left(v_{x}\right)=C e^{-\frac{m v_{x}^{2}}{2 k_{B} T}}
$$

where $g\left(v_{x}\right)$ is the probability density. In principle the velocity component $\left.v_{x} \in\right]-\infty, \infty[$, thus by directly integrating we find (Serway \& Jewett, 2014; Lemons, 2013):

$$
C=\left(\frac{m}{2 \pi k_{B} T}\right)^{1 / 2} \text {. }
$$


We have used the normalization condition, which requires that the sum of probabilities for all possible outcomes to be equal to unity. By substituting (4) into (3) we obtain

$g\left(v_{x}\right)=\left(\frac{m}{2 \pi k_{B} T}\right)^{1 / 2} e^{-\frac{m v_{x}^{2}}{2 k_{B} T}}$.

Clearly the theoretical distribution of molecules against $v_{x}$ for a one-dimensional ideal gas is Gaussian (Feller, 1957; Cox \& Miller, 1972; Huang, 1987; van Kampen, 2007; Lemons, 2013; Serway \& Jewett, 2014; Reichl, 2016). The same argument can be used even if we consider a onedimensional ideal gas in the $y$ and $z$ directions. In those cases, the density functions will be

$g\left(v_{y}\right)=\left(\frac{m}{2 \pi k_{B} T}\right)^{1 / 2} e^{-\frac{m v_{y}^{2}}{2 k_{B} T}}$.

$g\left(v_{z}\right)=\left(\frac{m}{2 \pi k_{B} T}\right)^{1 / 2} e^{-\frac{m v_{z}^{2}}{2 k_{B} T}}$

\section{Two-dimensional ideal gas}

The two-dimensional ideal gas is collection of molecules that move exclusively in two dimensions. Interestingly there are many works concerning this type of gas, despite its nonapplicability in real world problems (Ftačnik, et al., 1983; Bonomo \& Riggi, 1984; Aiello-Nicosia \& Sperandeo-Mineo, 1985; Bellomonte \& Sperandeo-Mineo, 1997). In such a system, the molecules move on a plane. Therefore, the velocity vector of each molecule is $\mathrm{v}=\left(v_{x}, v_{y}\right)$ as shown in Figure 1. The distribution of molecules against speed for each component is normal as in (5) for the $x$ component and $\left(5^{\prime}\right)$ for the $y$ component. Recall that the velocity components of molecules are independent from each-other. Therefore, the distribution for $v$ will be the product of the respective distribution of each component (Lemons, 2013; Reichl, 2016).

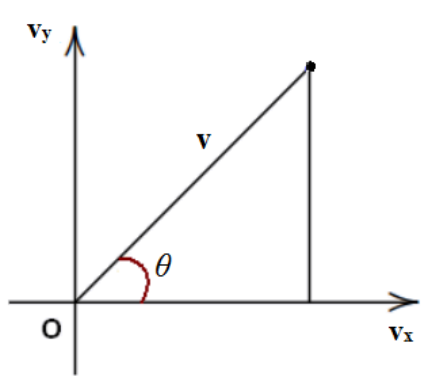

Figure 1. The transformation from Cartesian to the polar system in the velocity space.
In order to find the Maxwell's distribution in two dimensions, we should write for the probability density

$g\left(v_{x}, v_{y}\right) d v_{x} d v_{y}=C_{1} e^{-\frac{m v_{x}^{2}}{2 k_{B} T}} \times e^{-\frac{m v_{y}^{2}}{2 k_{B} T}} d v_{x} d v_{y}=C_{1} e^{-\frac{m\left(v_{x}^{2}+v_{y}^{2}\right)}{2 k_{B} T}} d v_{x} d v_{y}$.

It is extremely useful to switch from Cartesian to polar coordinates in the velocity space according to the transformations (see Figure 1 for reference)

$\left\{\begin{array}{l}v_{x}=v \cos \theta \\ v_{y}=v \sin \theta\end{array}\right.$.

Thus, the probability density function (6) can be written as

$g(v, \theta) d v d \theta=C_{1} v e^{-\frac{m v^{2}}{2 k_{B} T}} d v d \theta$

The arbitrary constant $C_{1}$ is gauged in a similar fashion as described in the previous paragraph, where the double integration is performed for both variables. The integration leads to (Lemons, 2013; Reichl, 2016):

$C_{1}=\frac{m}{2 \pi k_{B} T}$.

Thus, substituting (9) into (8) and integrating with respect to $\theta$, one obtains

$$
g(v)=\frac{m}{k_{B} T} v e^{-\frac{m v^{2}}{2 k_{B} T}} .
$$

The final result is obtained after integration with respect to $\theta$.

The shape of function (10) resembles quite a lot with the familiar Maxwell curve for three dimensions (Huang, 1987; van Kampen, 2007; Lemons, 2013; Reichl, 2016). The main difference is the exponent of the speed that is one in this case and two for the three-dimensional gas. An important result that we will use to make quantitative evaluations is the average speed, i.e. the first moment of the distribution (Huang, 1987; Serway \& Jewett, 2014; Reichl, 2016). This quantity can be evaluated by performing straightforward integration to be

$$
\langle v\rangle=\left(\frac{\pi k_{B} T}{2 m}\right)^{1 / 2},
$$


where $R$ is the gas constant, $M$ is the molar mass of the gas and $T$ is the thermodynamic temperature. From equation (11) we can express $\pi$ as

$\pi=\frac{2\langle v\rangle^{2}}{\left(\frac{k_{B} T}{m}\right)}$.

Therefore, if we know the average speed or the first moment of the distribution, we can theoretically obtain the value of $\pi$. The relation (12) resulted to be a useful measure to provide quantitative comparison between the simulated and theoretical distributions.

\section{Three-dimensional ideal gas}

The next ideal gas case is that in three dimensions. The molecules of the gas are free to move in space. The velocity vector for each molecule is the $\mathbf{v}=\left(v_{x}, v_{y}, v_{v}\right)$ as shown in Figure 2, left panel. Again the distribution of molecules for each component is normal as in (5), (5') and (5"). Naturally the velocity components of the molecules are independent the distribution for the speed $v$ will be evaluated in analogy with (6). Therefore, the probability density for the three-dimensional ideal gas is (Serway \& Jewett, 2014; Lemons, 2013)

$$
g\left(v_{x}, v_{y}, v_{z}\right) d v_{x} d v_{y} d v_{z}=C_{2} e^{-\frac{m\left(v_{x}^{2}+v_{y}^{2}+v_{z}^{2}\right)}{2 k_{B} T}} d v_{x} d v_{y} d v_{z} .
$$

Similarly as for the two-dimensional gas, we will switch from the Cartesian to the spherical polar coordinates in the velocity space according to the transformations (Figure 2)

$$
\left\{\begin{array}{l}
v_{x}=v \cos \varphi \sin \theta \\
v_{y}=v \sin \varphi \sin \theta . \\
v_{z}=v \cos \theta
\end{array}\right.
$$

When performing such transformation, one should consider how this very procedure affects the infinitesimally small volume of space, which in this case becomes (Figure 2, right panel)

$$
d v_{x} d v_{y} d v_{z}=v^{2} \sin \theta d v d \theta d \varphi
$$

being very similar to the respective transformation between Cartesian and spherical polar coordinates in position space. By substitution of (14) and (15) into (13) and then integrating accordingly, we obtain

$C_{2}=\left(\frac{m}{2 \pi k_{B} T}\right)^{3 / 2}$.
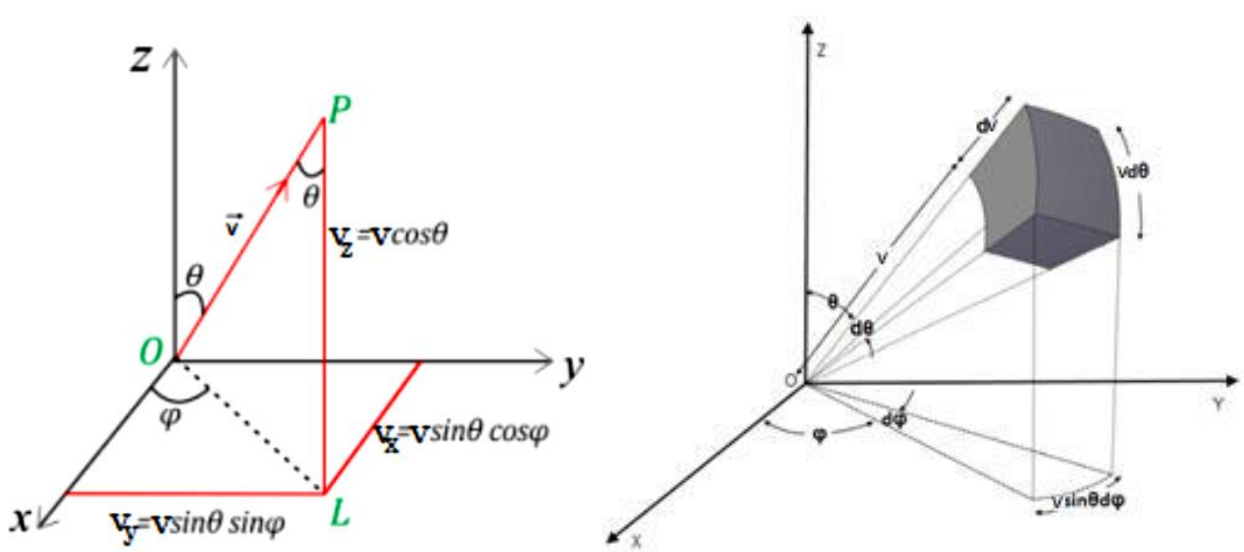

Figure 2. The transformation from Cartesian to the spherical polar system in the velocity space. In the left panel is shown schematically the relation between components in both coordinate systems. In the right panel is shown the elementary volume in both coordinate systems. The figures can be found freely on the internet.

The probability density (13) consequently can be casted into

$g(v)=4 \pi\left(\frac{m}{2 \pi k_{B} T}\right)^{3 / 2} v^{2} e^{-\frac{m v^{2}}{2 k_{B} T}}$

The final result is derived after integrating with respect to $\theta$ and $\varphi$.

A helpful result is again the expression of the average speed. In three dimensions, the average speed reads

$\langle v\rangle=\left(\frac{8 k_{B} T}{\pi m}\right)^{1 / 2}$.

Isolating $\pi$ on the left side, we can finally write

$\pi=\frac{8\left(\frac{k_{B} T}{m}\right)}{\langle v\rangle^{2}}$. 


\section{Numerical method}

\section{The central limit theorem}

The central limit theorem (CLT) is one of the crucial results in probability theory (Feller, 1957; Cox \& Miller, 1972). Actually the work described in the actual paper is a demonstration of this theorem. Let us suppose that we have a set of independent identically distributed random variables $X_{i}$. The expected value $\mu$ and the variance $\sigma^{2}$ of the distribution are finite. We construct the variable

$S_{n}=X_{1}+X_{2}+\mathrm{K}+X_{n}$,

where $n$ is the number of added random variables. Next we define a new variable

$Z_{n}=\frac{S_{n}-n \mu}{\sqrt{n \sigma^{2}}}$.

The standard deviation is about the initial set of random numbers. Then for every real $a$ and $b$, such that $a<b$, it is valid

$\lim _{n \rightarrow \infty} P\left(a<Z_{n}<b\right)=\frac{1}{\sqrt{2 \pi}} \int_{a}^{b} e^{\frac{x^{2}}{2}} d x$.

Equation (22) constitutes one of the formulations of the CLT (Feller, 1957).

We stated nothing regarding the nature of the distribution of the original variables $X_{i}$. Actually it is not relevant if the variable is discrete or continuous. The result (22) will be valid as long as the expectation and variance are defined (Feller, 1957; Cox \& Miller, 1972). In Figure 3 is shown a typical example that verifies the CLT. The uniformly distributed random variables $X_{i}$ are generated independently from each other. We add a different number of them $n$. It is clear that as $n$ becomes increasingly larger the PDF of the $Z_{n}$ variables approaches the PDF of the normal distribution.

$$
Z_{1}=\frac{X_{1}-\frac{1}{2}}{\sqrt{\frac{1}{12}}}
$$

PDF of $Z_{1}$

Assumptions:

- $X_{1}, X_{2} \ldots$ are iid Uniform $(0,1)$

- $Z_{n}=\frac{X_{1}+X_{2}+\ldots+X_{n}-\frac{n}{2}}{\sqrt{\frac{n}{12}}}$

$Z_{2}=\frac{X_{1}+X_{2}-1}{\sqrt{\frac{2}{12}}}$

PDF of $Z_{2}$

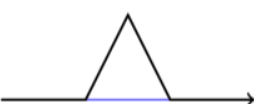

$Z_{3}=\frac{X_{1}+X_{2}+X_{3}-\frac{3}{2}}{\sqrt{\frac{3}{12}}}$

PDF of $Z_{3}$

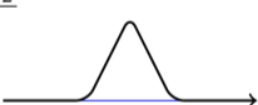

$Z_{30}=\frac{\sum_{i=1}^{30} X_{i}-\frac{30}{2}}{\sqrt{\frac{30}{12}}}$

PDF of $Z_{30}$

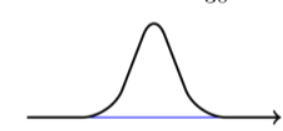

Figure 3. Schematic representation of the CLT. The independent random variables satisfy the uniform distribution. On the right is shown what happens to the PDF of the $Z$ variable when the number of $X i$ variables increases. When adding 30 Xi's the PDF of the $Z$ variable resembles the normal distribution. The distribution becomes normal as the number of added Xi's is infinite. The picture is reproduced from (Feller, 1957; Cox \& Miller, 1972).

\section{Simulating the ideal gas}

One of the typical applications of CLT is related to coin tosses. In such case, one associates the value 1 if head is tossed and -1 if tail is tossed. We generate uniformly distributed random numbers that fall in the open interval $] 0,1[$. Then we transform these numbers into a set of integers $X_{i} \in\{-1,1\}$. Afterwards, we construct with these numbers the following quantity:

$$
v_{x}=X_{1}+X_{2}+\ldots+X_{n} \text {. }
$$

Due to the combination 1 and -1 , the quantity (23) can have positive, negative or zero value. Thus, it becomes very handy to simulate the velocity component (in 1D). Furthermore, the random nature of the numbers in (23) ensures the randomness of the velocity component. In this 
way we can simulate the velocities of the molecules of a given gas in a given temperature. The uniform distribution of the $X_{i}^{\prime}$ s is characterized by (Boriçi, 2019):

$\mu=E[X]=0, \quad \sigma^{2}=E\left[X^{2}\right]=1$.

Thus, according to the requirements of the CLT, we construct the normalized sums:

$Z_{n}=\frac{v_{x}}{\sqrt{n}}$.

We square up equation (25) and find that

$Z_{n}^{2}=\frac{v_{x}^{2}}{n}$

Taking into consideration the Maxwell's distribution PDF in (5), we can deduce that the variance of the distribution is

$$
\sigma_{v}^{2}=\frac{k_{B} T}{m} \text {. }
$$

The construction (26) indicates that we should set $n=\sigma_{v}^{2}$.

Thus, the parameter $n$ is the square of the standard deviation of the limiting normal distribution in (22). Clearly $n$ depends on the thermodynamic temperature $T$; we consider this parameter as the model's temperature. By substituting the values of the Boltzmann constant, the average molecular mass of the air molecules and temperature $300 \mathrm{~K}$, one finds $n=86,000$. In the next section we never analyze such a value, because it requires relatively extended computational resources. However, to show the efficiency of our approach, it is not necessary to employ such large values for $n$. The other parameter in our algorithm is the number of molecules $N$.

In order to simulate the distribution of velocities for a given number $N$ of molecules, whatever the dimensionality, we initially generate an $n \times N$ matrix with uniformly distributed random numbers 1 and -1 . Thus, the velocity component for each molecule is found by simply adding the random values in each column. The numbers of rows coincide with the model temperature. This allows us to control the temperature of the gas and analyze its effect on the distribution. Intuitively, the greater the temperature the more disparate the velocity values are and vice versa. Then we construct histograms of the velocity component or speed where the height of a column measures the empirical probability for the corresponding interval. Thus, we obtain the simulated discrete PDF that is then compared with the respective theoretical PDF. This algorithm can be easily implemented in the Matlab/Octave platforms. The codes with detailed comments can be found in the Appendix section.

\section{Results}

\section{One-dimensional ideal gas}

Let we report initially the results from the simulations of the Maxwell's distribution for the onedimensional ideal gas. The respective code is shown in the Appendix section. In all the distributions shown in Figure 4, the model temperature is $n=300$. The conversion to the thermodynamic temperature scale can be done easily by knowing the molar mass of a given gas. However we should be careful with the chosen value of $n$ in accordance to the computational limitations of the computer used for the simulations.
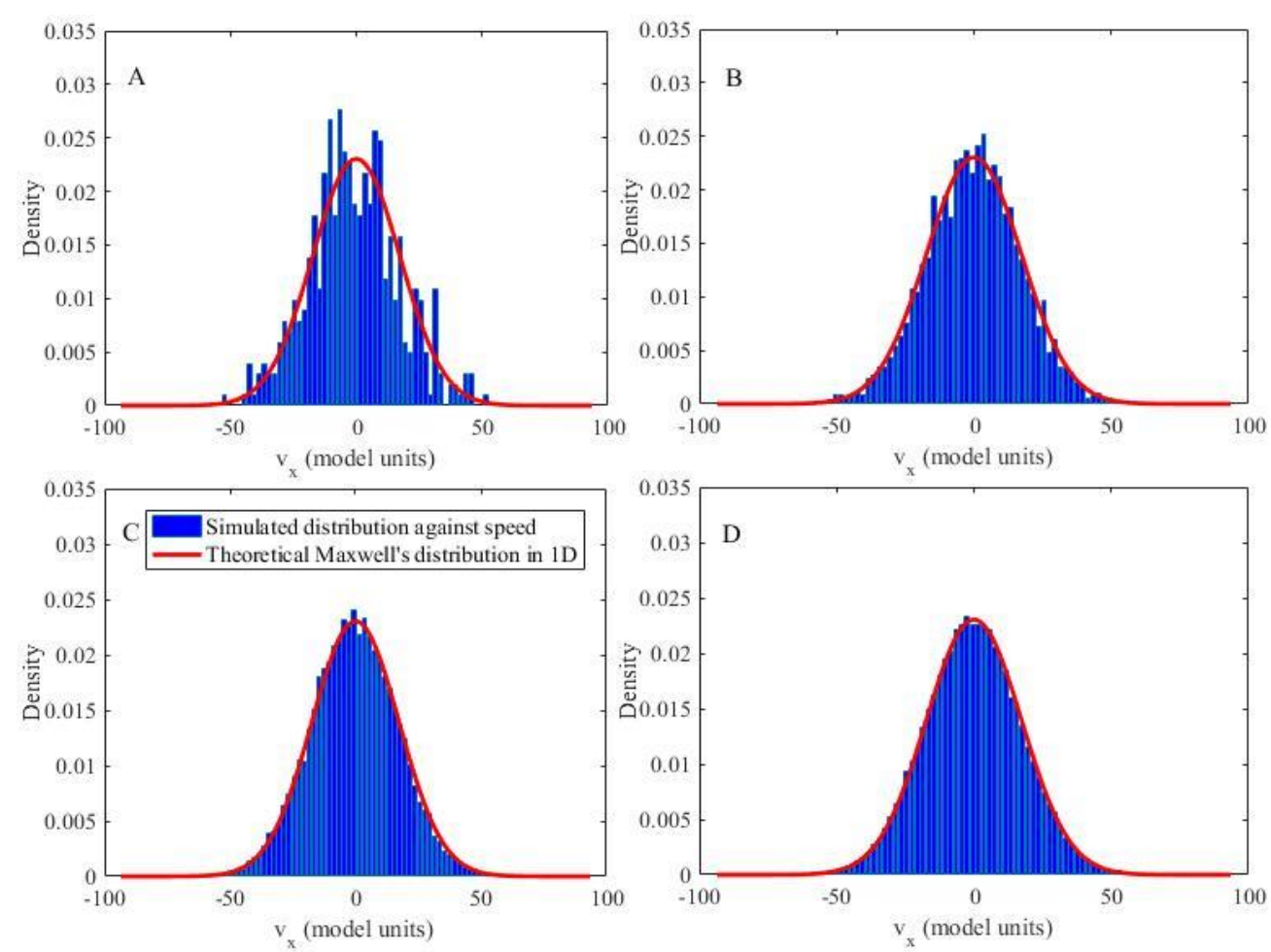

Figure 4. Here are shown the Maxwell's distribution for the one-dimensional ideal gas for different number of molecules. The model temperature is the same $n=300$. The number of molecules is 500 (panel A), 5000 (panel B), 20000 (panel C) and 60000 (panel D). The velocity component magnitude is extended to 5.5 times the standard deviation. The red curve represents the theoretical Maxwell's distribution for one dimension. The legend shown in panel $C$ is the same for all the other panels as well. 
Visually it is quite easy to determine which simulations are quite close to the theoretical Maxwell's distribution and which are not. For instance, the simulations in panel C and D (Figure 4) are quite close to the theoretical curve compared to the simulations shown in panels $\mathrm{A}$ and $\mathrm{B}$. However, there is needed to be used a quantitative test to better evaluate the outcome of the simulation. The Maxwell's distribution for the one-dimensional gas is simply a normal distribution. The area under the PDF curve for a given interval represents the probability for the variable to be in that interval. Also, it is a well-known fact that the probability for the variable to be in the interval $[\mu-\sigma, \mu+\sigma]$, where $\mu$ and $\sigma$ are respectively the mathematical expectations and standard deviation of the distribution (Feller, 1957; Boriçi, 2019), is $68.27 \%$. Recall that $\sigma=\sqrt{n}$

. The same argument can be used for the intervals $[\mu-2 \sigma, \mu+2 \sigma]$ and $[\mu-3 \sigma, \mu+3 \sigma]$ as well. For these intervals the respective areas under the curve are respectively 0.9545 and 0.9973 . We can use these facts to analyze the nature of the simulated distributions.

The area under the distribution in panel A for the interval $[\mu-\sigma, \mu+\sigma]$ is 0.7000 or $70.00 \%$. The probabilities for the other distributions are respectively $64.56 \%, 67.46 \%$ and $67.42 \%$. In the first simulation we can see that more molecules are concentrated in the central hill of the distributions, while further away from the symmetry axis the number of molecules having the respective speeds is notably smaller that in theory. This explains the higher probability found. The opposite is observed in the second simulation where the central hill is lower than the theoretical prediction. For the other simulations the variations are minimal. The discussed results are for the specific simulated distributions shown in Figure 4. For another set of simulations the results would be different; after all there are involved random numbers. In order to obtain more robust results, there should be analyzed many simulations for one combination of $n$ and $N$, something that can be left as a work for the student.

A rather interesting result of the simulations is the ability to model the effect the change in temperature has on the distribution. The greater the temperature is the greater the average kinetic energy of the molecules. Hence, the fraction of molecules having larger velocities is higher the higher the temperature. As a consequence the theoretical curve should widen and at the same time the central hill should become lower (Figure 5). Mathematically the larger the temperature is the larger the standard deviation is, hence $n$ is larger. Therefore, the results shown in Figure 4 are pretty much expectable. However, the real motivating results are found for the simulations of the ideal gas two and three dimensional where the effect of temperature on the distribution is reproduced effectively

Before we proceed to the next paragraphs, we should make an important remark regarding the nature of the simulations shown in this paper. They do not present the thermodynamic evolution of the system. The ideal gas does not start in a given initial state out of equilibrium and we do not follow its evolution toward this state. The ideal gas is already in the thermodynamic equilibrium state and it possesses the temperature $T$. Clearly the approach discussed in this paper works well for a relatively large number of molecules $N$. However, due to computational confinements $N$ cannot take an unlimited value and it depends on the properties of the computer. In spite of such limitations, we have seen from many simulations that for $N>10,000$ the simulated distributions are reasonably close to the theoretical curve. This value of $N$ is far away from the actual number of molecules even in a small quantity of gas and yet the distribution of molecules versus the speed $v_{x}$ is already very close to the theoretical Maxwell's distribution. This very fact provides evidence in support of the accuracy of the assumptions that led to the distribution formula.

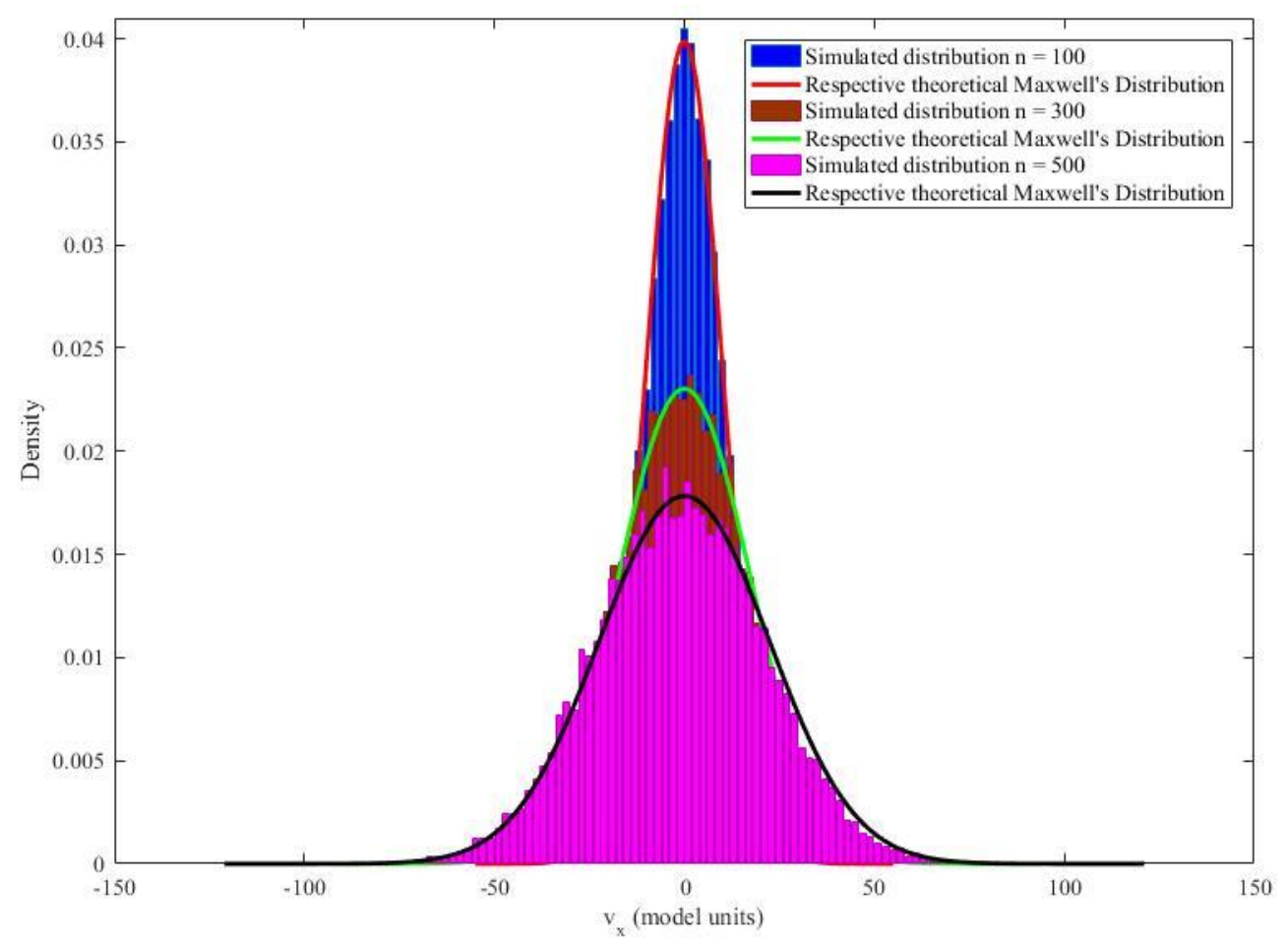

Figure 5. Maxwell's distribution for the one-dimensional ideal gas for different model temperatures The number of molecules in each case is $N=20,000$. The model temperature is 100 (red curve), 300 (green curve) and 500 (black curve). The velocity component magnitude is extended to 5.5 times the standard deviation. The curves represent the theoretical Maxwell's distribution for one dimension in each case.

\section{IJPCE - International Journal of Physics and Chemistry Education, 13(2), 33-45}


Two-dimensional ideal gas

The procedure followed for the simulation of Maxwell's distribution for the one-dimensional ideal gas is fundamental when increasing the dimensionality of the system. This fact can be seen immediately in the codes used for the ideal gas in two (the actual paragraph) and three dimensions (next paragraph).

In Figure 6 are shown four typical simulations of the two-dimensional ideal gas. The number of molecules in each case is 500, 5000, 10000 and 50000 and the model temperature is the same $n=300$. Interestingly, the simulated distributions do not appear to be very different from each other and the increase in $N$ seems virtually irrelevant in the appearance of the histogram. Actually it seems to be a specific feature for this dimensionality. For instance, the simulated distributions for the one- and three-dimensional cases are visibly different.
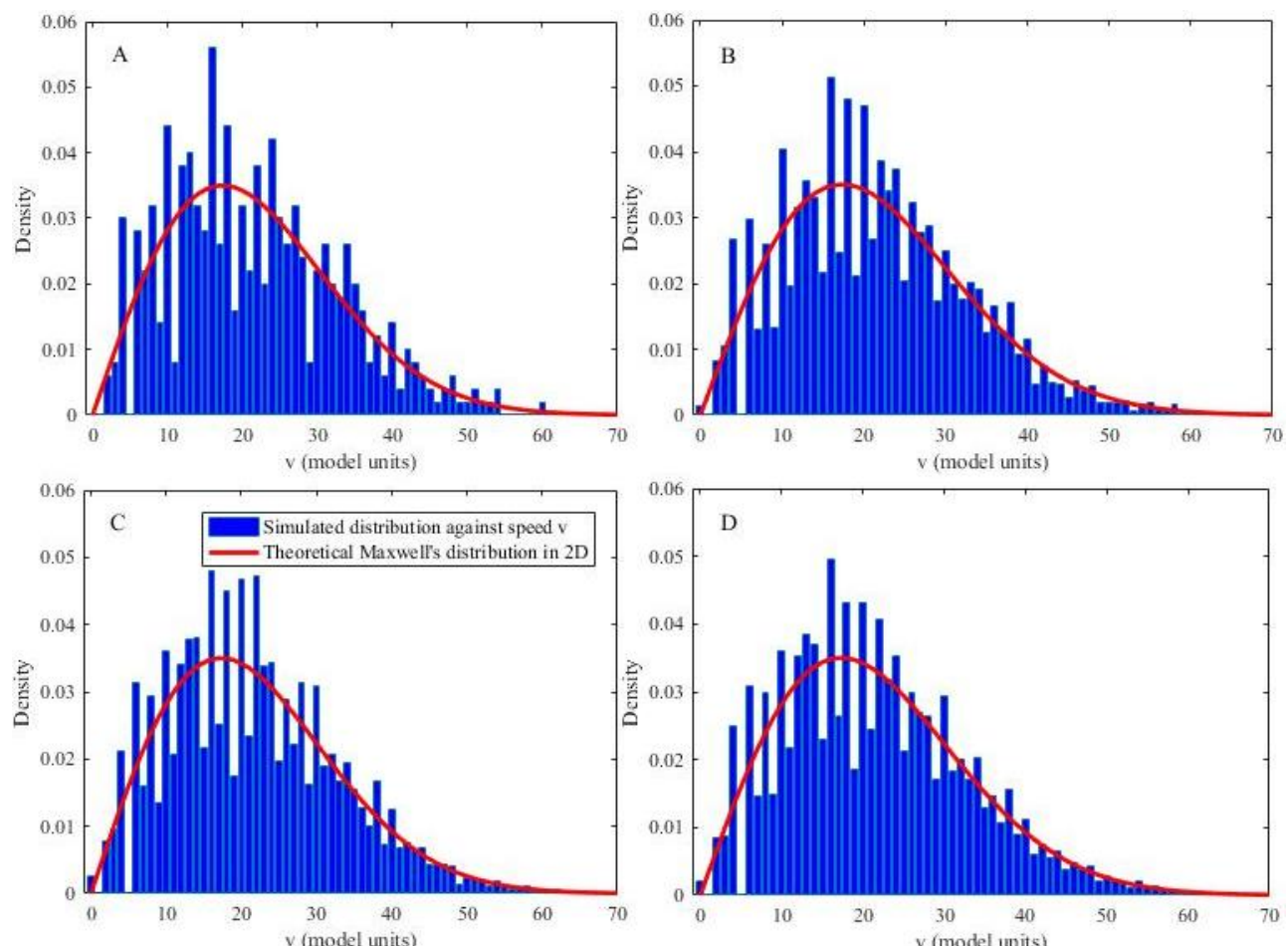

Figure 6. Maxwell's distribution for the two-dimensional ideal gas for different number of molecules. The model temperature is the same $n=300$. The number of molecules is 500 (panel A), 5000 (panel B), 10000 (panel C) and 50000 (panel D). The speed magnitude is extended to 5.5 times the standard deviation. The red curve represents the theoretical Maxwell's distribution for two dimensions. The legend shown in panel $\mathrm{C}$ is the same for all the other panels as well.
In order to better analyze the nature of each simulated distribution, we need to describe a procedure that can provide quantitative results. This procedure is based on formulae (12) and (20) for the 3D gas; these expressions relate the average speed with the model temperature $n$. to find the average speed we exploit the discrete nature of the simulated distributions. For instance, the simulated distribution actually is a discrete probability distribution. The number in the $x$ axis pertains to the speed in model units, while the height of the column corresponds to the empirical probability for each value of the speed. The average speed is then found straightforwardly. Afterwards we use the value found to evaluate the empirical values of $\pi$, following (12). The closer this empirical value is to $\pi$, the closer the simulated distribution is considered to be to the theoretical Maxwell's distribution.

We have evaluated the empirical value of $\pi$ for each simulated distribution shown in Figure 6 . For the distribution in panel A the difference between the empirical and the theoretical value of $\pi$ is $1.50 \%$. For the simulated distributions shown in the subsequent panels, the difference is respectively $1.26 \%, 0.77 \%$ and $0.06 \%$. Obviously the increase of the number of molecules $N$ will decrease the difference between the empirical and theoretical values of $\pi$. Thus, despite the visual similarities between the simulated distributions, the quantitative procedure we followed distinguishes them pretty clearly. Similarly as for the one-dimensional case, the values of difference found are for the shown distributions. In order to have a far more robust evaluation, the student has to perform many simulations, calculate the relative difference for each case and then find the average value together with the respective error. This process can be assigned to students as an effective class work in order to increase their understanding about computer generated random numbers and how to interpret the simulations based on them.

The effect of temperature on the simulated distributions is investigated as well and they are shown in Figure 7. The red curve pertains to the case with model temperature $n=100$, the green curve to $n=300$ and the black curve to $n=500$. A higher temperature means a greater fraction of molecules with larges kinetic energy. Therefore the peak of the distributions should move toward higher speeds. Meanwhile, due to the constant number of molecules in the ideal gas, the height of the distribution should be lower. Mathematically, the area under the curve should remain constant and the changes that the curve is subjected to make possible such feature. Therefore, the approach followed by us results to be effective in reproducing this important feature of Maxwell's distribution. 


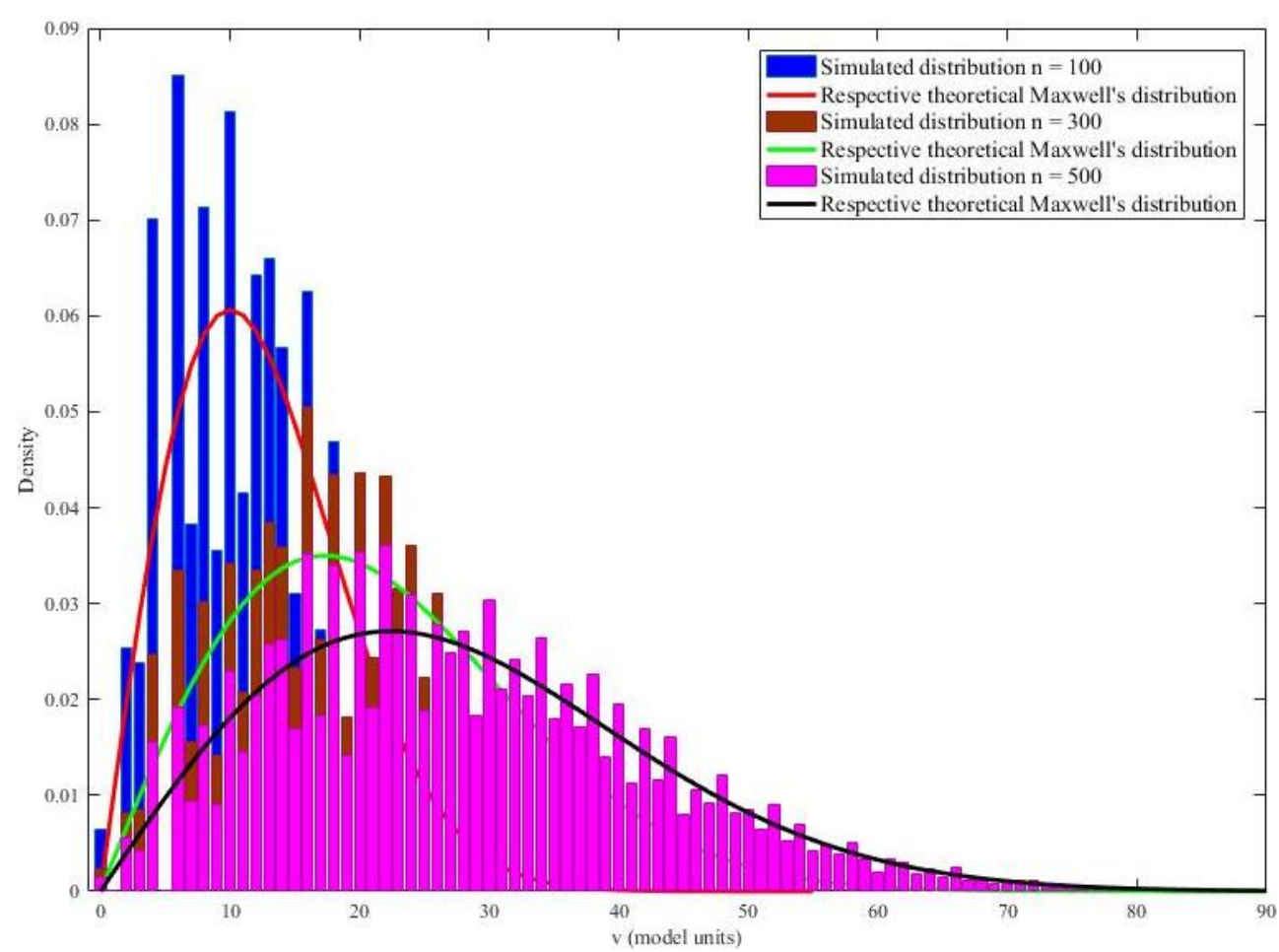

Figure 7. Maxwell's distribution for the two-dimensional ideal gas for different model temperatures. The number of molecules in each case is $\mathrm{N}=25,000$. The model temperature is 100 (red curve), 300 (green curve) and 500 (black curve). The speed magnitude is extended to 5.5 times the standard deviation. The curves represent the theoretical Maxwell's distribution for two dimensions in each case.

\section{Three-dimensional ideal gas}

We follow a very similar procedure to the three-dimensional gas. The simulated distributions are shown in Figure 8. The number of molecules in each case is 500, 5000, 10000 and 25000. One can choose higher values of $N$, but needs to use sources with higher computational power. Again the model temperature is the same for all simulations and is $n=300$. Differently from the 2D case, the simulated distributions show a qualitative visual improvement as $N$ increases.

The quantitative procedure explained in the previous paragraph is based on expression (20) for the 3D gas. Again we have calculated the relative difference between the empirical, associated with the distribution, and theoretical value of $\pi$ for each simulated distribution. The differences are respectively (starting from panel A) $0.81 \%, 0.71 \%, 0.18 \%$ and $0.06 \%$. Similarly as for the
2D gas, the increase of the number of molecules $N$ decreases the relative difference between the empirical and theoretical values of $\pi$, as expected. However, it is noticeable that the relative change is usually smaller for the $3 \mathrm{D}$ gas than for the $2 \mathrm{D}$ gas simulations. This is related to the fact that we use consistently more computer generated random numbers in the former case. Again, the values of relative difference found pertain to the specific simulated distributions shown in Figure 8. Therefore, there is needed to perform numerous simulations, find the relative difference for each of them and afterward calculate the average of all the found values and the respective error.
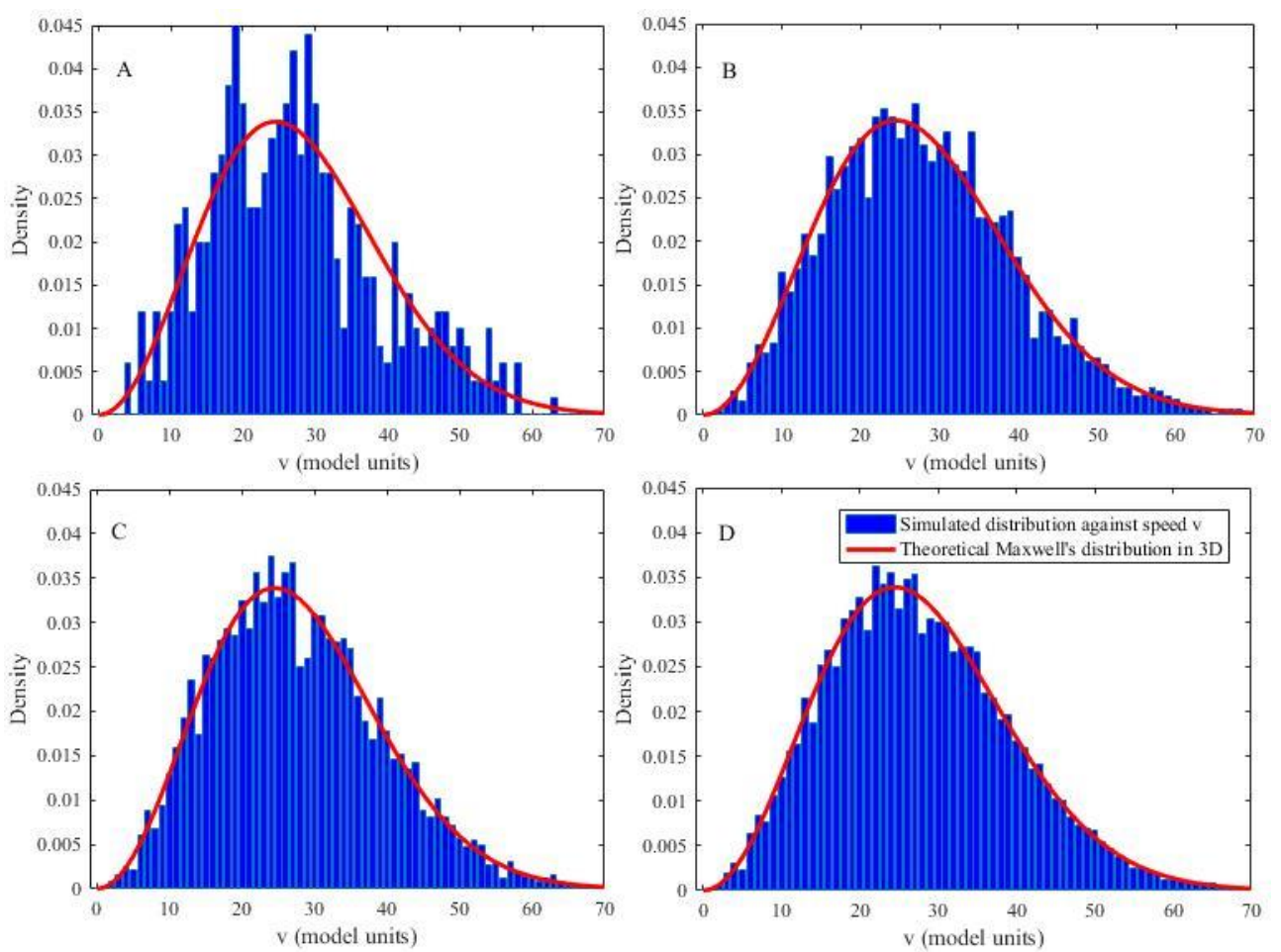

Figure 8. Maxwell's distribution for the three-dimensional ideal gas for different number of molecules. The model temperature is the same $n=300$. The number of molecules is 500 (panel $A$ ), 5000 (panel B), 10000 (panel C) and 50000 (panel D). The speed magnitude is extended to 5.5 times the standard deviation. The red curve represents the theoretical Maxwell's distribution for two dimensions. The legend shown in panel $\mathrm{C}$ is the same for all the other panels as well. 
As in the previous paragraphs, we investigate the effect the change in temperature has on the simulated distributions (Figure 9). The number of molecules is fixed at 25,000, while the model temperatures are $n=50$ for the red curve, $n=150$ for the green curve and $n=400$ for the black curve. Again we mention that all the combinations shown here are for practical demonstrations and no specific real world situations is associated to them. As for the $2 \mathrm{D}$ gas, the higher the temperature, the lower the peak and the more it is shifted toward the higher speeds.

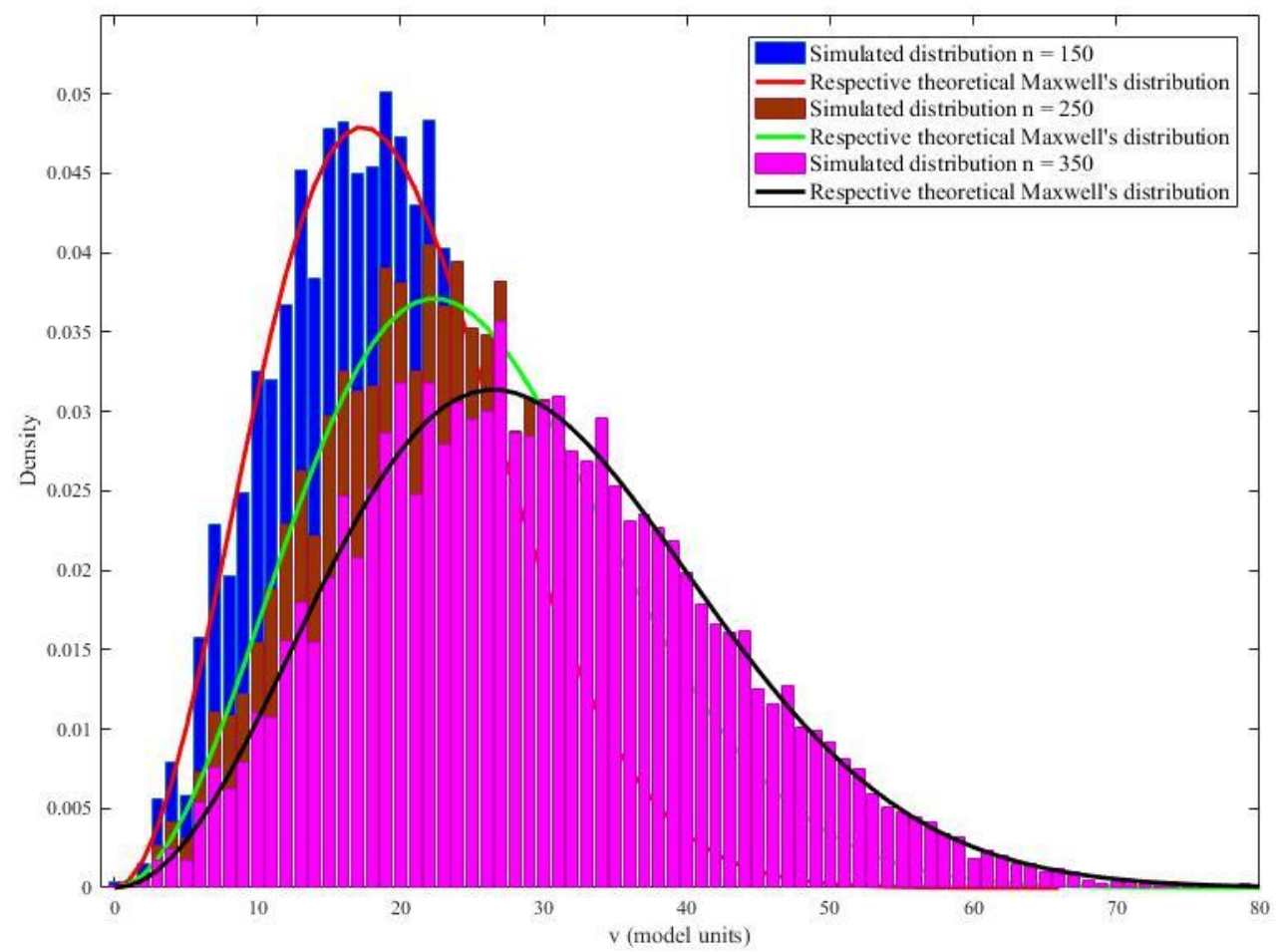

Figure 9. Here are shown the Maxwell's distribution for the three-dimensional ideal gas for different model temperatures. The number of molecules in each case is $\mathrm{N}=25,000$. The model temperature is 150 (red curve), 250 (green curve) and 350 (black curve). The speed magnitude is extended to 5.5 times the standard deviation. The curves represent the theoretical Maxwell's distribution for three dimensions in each case.

The simulated distributions for the two- and three-dimensional ideal gas appear to be very similar at first sight. However, this is not the case as illustrated in Figure 10. For both simulations the number of molecules is $N=25,000$ and the model temperature is $n=300$. The distribution for the $3 \mathrm{D}$ gas is shifted on the right, toward higher speeds and the respective peak is lower. The molecules of three-dimensional gas have three degrees of freedom compared to the two degrees of freedom of the 2D gas. Therefore, the molecules of the 3D gas possess on average more kinetic energy than the molecules in two dimensions. This explains why more molecules in $3 \mathrm{D}$ have higher speeds and why the distribution is shifted on the right.

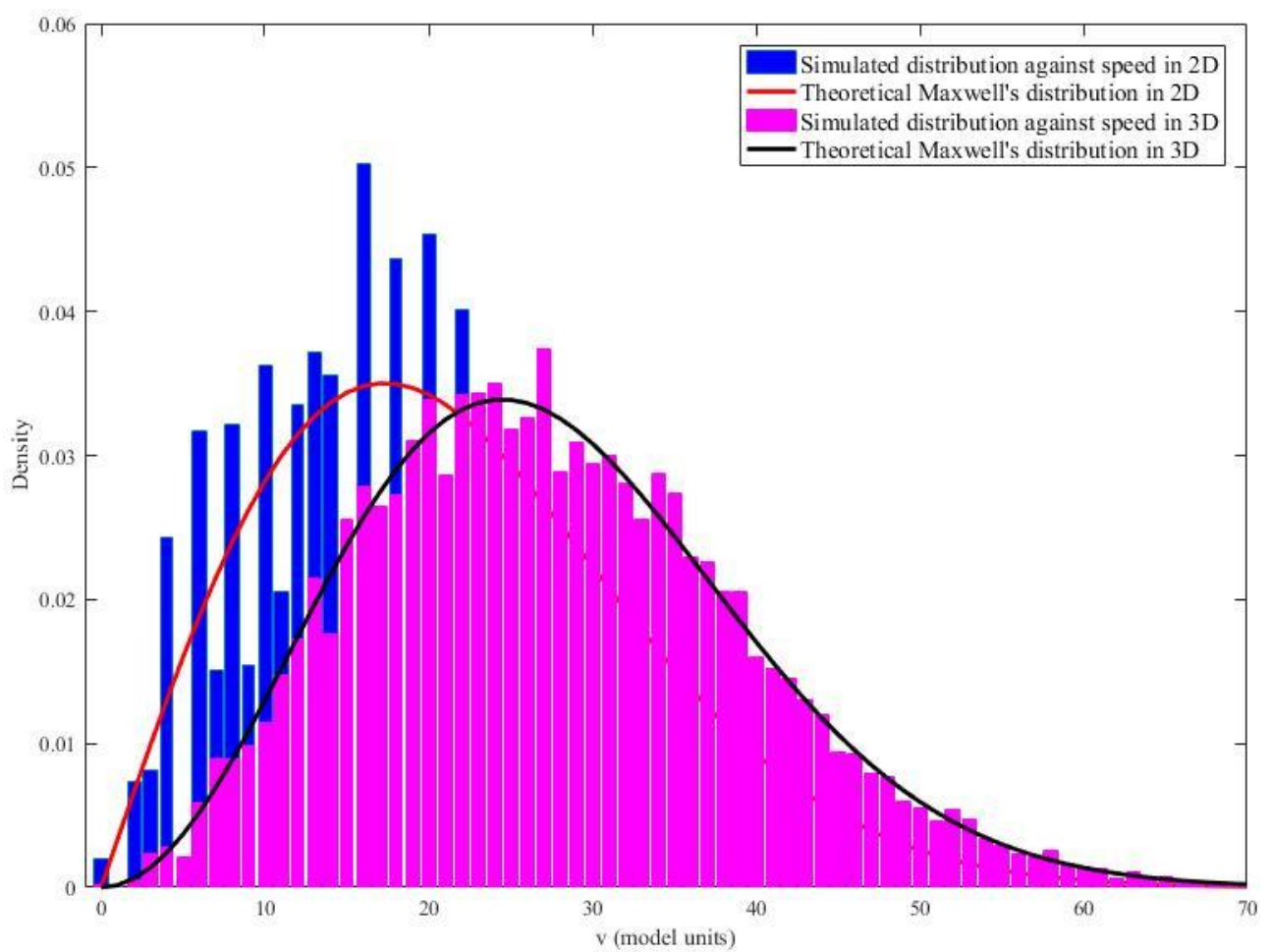

Figure 10. Maxwell's distribution for the two- and three-dimensional ideal gas. In both cases the model temperature is $n=300$ and the number of molecules is $N=25,000$. The speed magnitude is extended to 5.5 times the standard deviation. The curves in solid line represent the theoretical Maxwell's distribution for each case.

\section{Pedagogical discussion}

The material discussed thus far can be easily implemented in teaching activities. In an introductory physics course the mathematical aspects of the Maxwell's distribution may be omitted and usually only the distribution itself is discussed. However, even if the mathematics is carried out extensively, the concept of probability density function or independence against orientation in space are not that easy to grasp by students. Following the algorithm discussed 
here, the lecturer can discuss the ease the underlying physical assumptions that lead to Maxwell's distribution, by even omitting the tedious mathematical calculations.

The initial steps of the algorithm (see Appendix for details) describe how to simulate the different speeds of the molecules/atoms, thus incorporating the assumption that different molecules move with different speed. By simulating different components of the velocity, one for each dimension, it becomes clear to the students that each of the components are statistically equivalent and have the same properties. Finally, by merging them together, we obtain the simulated distribution.

This numerical procedure emphasizes some crucial aspects of Maxwell's distribution. It becomes clear to the students that distributions are statistical in nature and that to be observed the physical system needs to have many constituents (atoms or molecules). This is evident in Figures 4, 6 and $\mathbf{8}$, where by increasing the number of molecules, the simulated distribution is much closer to the theoretical counterpart. Furthermore, the simulations provide a statistical meaning for quantities like average speed, most probable speed or root mean square speed. Lastly, the simulations make the spatial isotropy tangible to the students.

We should remark that the material presented in the actual paper is not intended to replace the theoretical discussion. Actually, we suggest this approach to be used in one or two lecture classes in Thermodynamics classes to show how the Maxwell's distribution is natural in a system like the ideal gas.

\section{Discussions and conclusions}

In the present paper we discuss an interesting approach to study the Maxwell's distribution of velocities for the ideal gas in a pedagogically effectively manner. Quite interestingly the numerical approach is helpful in simplifying the theoretical exposition. Thus, high school students can be extensively presented with such an advanced topic omitting the rather complicated mathematical operations.

The problem of simulating the Maxwell (- Boltzmann) distribution is not recent. There have been several papers in the past dealing with various numerical aspects (Ftačnik, et al., 1983; Bonomo \& Riggi, 1984; Aiello-Nicosia \& Sperandeo-Mineo, 1985; Bellomonte \& SperandeoMineo, 1997; Jameson \& Brüschweiler, 2020). Some of them deal with the two-dimensional ideal gas, where the molecules are considered as rigid disks all identical (Ftačnik, et al., 1983; Bonomo \& Riggi, 1984; Aiello-Nicosia \& Sperandeo-Mineo, 1985). In these papers the authors analyze the system from a kinetic perspective, by studying how the system evolves while the molecules collide elastically in the Newtonian mechanics framework. Interestingly their motivations was the same as the one expressed in this paper: simplify the introduction of students to this specific topic by avoiding complicated mathematics and using computers to achieve the goal.
The authors perform the simulations by considering an initial random positions and velocities and then simulate the collisions between them. The authors analyze snapshots at different subsequent times. That is different from our approach where we analyze a single snapshot of an ideal gas already in equilibrium. The collisions are simulated by using an interaction matrix that relates relative velocities before and after the collision event. Also the scattering angles are assumed to be uniformly distributed (Ftačnik, et al., 1983; Bonomo \& Riggi, 1984). A slightly different approach would be to start with randomly distributed molecules with random uniform velocities and let the system evolve with time. In every can be calculated the Boltzmann's constant $H$ so that one can determine how close the system is to equilibrium (Aiello-Nicosia \& SperandeoMineo, 1985). Ftačnik, et al., 1983, perform simulations with 5, 10 and 50 molecules and report in Figure 2 in their paper 5,000 snapshots. This would be the same as having one snapshot of $25,000,50,000$ and 250,000 molecules respectively. This is in agreement with our results because we observe that the numerical distribution is very close to the theoretical one once the number of molecules $N>20,000$

It is observed that the exact form of the initial distribution of velocities does not matter later in time when the system approaches equilibrium (Bonomo \& Riggi, 1984; Aiello-Nicosia \& Sperandeo-Mineo, 1985). Apparently the Maxwell distribution is just another manifestation of the Second Law of Thermodynamics and it can be proven numerically as well (Bellomonte \& Sperandeo-Mineo, 1997). A more statistical approach is followed recently (Jameson \& Brüschweiler, 2020) where the authors obtain the Boltzmann probability law, a special case of which is the Maxwell distribution law, just by allowing the gas to evolve to equilibrium from some random initial conditions. In all the above-mentioned studies, the gas evolvers toward the equilibrium state. The kinetic mechanism consider makes the simulations more challenging and the codes used more complicated for students. Our approach instead considers only equilibrium and is based on the much simpler notion of uniformly distributed random numbers. Thus, the codes are much easier and can be readily used by students even in high school.

The simulations in the present paper capture interesting features of the Maxwell's distribution for different dimensionalities. The most important aspect is that by increasing the number of molecules in the simulation, the closer the simulated and theoretical distributions are. Ideally the complete fit happens when the number of molecules goes to infinity. In practice, it suffices that the number of molecules $N>20,000$. However by increasing the computational capabilities, one can obtain a better between simulated and theoretical distributions.

The effect of temperature on the shape and peak location of the distribution is captured excellently by the actual numerical approach. The increase of the model temperature $n$, correctly displaces the peak of simulated distribution toward the higher velocities, i.e. higher energies, coinciding with the theoretical considerations. In order to perform simulations for ideal gas in room temperature, $n$ should be as high as 86,000 . Considering the numerical algorithm explained 
in section 3.2, the generated matrix should have large dimensions. This can be easily performed in powerful computer. However it is not necessary in confirming the effectiveness of the actual approach because the required values of $n$ and $N$ are much lower.

In order to compare quantitatively the simulated and theoretical distributions, we exploited the average speeds formulas. We isolated $\pi$ in each case and then we evaluated the empirical value of $\pi$ for each simulation in two and three dimensions. Generally we observed that by increasing the number of molecules the relative difference between the theoretical and empirical $\pi$ values becomes increasingly smaller. Thus, we can use the simulations as a numerical experiment to actually obtain approximations of $\pi$.

In this paper we analyzed the distribution for the ideal gas, which molecules possess only kinetic energy. It is rather interesting to analyze the gas in the homogeneous gravitations field, where the molecules possess also potential energy. There are works published in this direction that obtain the barometric formula for a gas composed of colliding massive points. The simulations actually demonstrate how the system evolves from a cluster of uniformly distributed molecules to a stratified one according to the barometric formula (Pantellini, 2000). This work is performed in the framework of the kinetic theory. We will analyze the barometric formula with our approach of the gas in equilibrium. Again, the aim will not be to show how the gas state evolves through time, but to directly analyze the equilibrium state. Thus, we will consider this more realistic physical system in a forthcoming paper.

We believe that numerical algorithms should become an integral part of teaching physics. They make difficult topics easier to understand and emphasize crucial aspects. Furthermore, they show students there are alternative ways of studying physical phenomena.

\section{Acknowledgments}

We would like to express our gratitude to the anonymous reviewers for their helpful suggestions that greatly improved the present paper.

\section{References}

Aiello-Nicosia, M.L., \& Sperandeo-Mineo, R. M. (1985). Computer simulation of a two-dimensional ideal gas: a simple molecular dynamics method for teaching purposes. European Journal of Physics, 6, 148-153.

Behringer E., \& Engelhardt L. (2017). Guest editorial: AAPT Recommendations for computational physics in the undergraduate physics curriculum, and the Partnership for Integrating Computation into Undergraduate Physics. American Journal of Physics. 85, 325-6.

Bellomonte, L., \& Sperandeo-Mineo, R. M. (1997). A statistical approach to the second law of thermodynamics using a computer simulation. European Journal of Physics, 18 (1997) 321-326.

Bonomo, R. P., \& Riggi, F. (1984). The evolution of the speed distribution for a two-dimensional ideal gas: A computer simulation. American Journal of Physics, 52, 54; http://doi.org/10.1119/1.13809

Boriçi A. (2019). Fizika me anë të programimit në Octavë dhe C++. Lecture notes in Albanian.

Cox D. R., \& Miller H. D. (1972). The theory of Stochastic Processes. Chapman and Hall, London, United Kingdom.
Daineko Y., Dmitriyev V., \& Ipalakova, M. (2017). Using virtual laboratories in teaching natural sciences: An example of physics courses in university. Comput. Appl. Eng. Educ. 25, 39-47.

Feller W. (1957). An Introduction to Probability Theory and its Applications, Vol. I, $2^{\text {nd }}$ edition. John Wiley \& Sons, New York, United states.

Ftačnik, J., Lichard, P., \& Pišut, J. (1983). A simple computer simulation of molecular collisions leading to Maxwell distribution. European Journal of Physics, 4, 68-71.

Huang, K. (1987). Statistical Mechanics, 2nd edition. John Wiley \& sons. New York, United states.

Jameson, G., \& Brüschweiler, R. (2020). Active Learning Approach for an Intuitive Understanding of the Boltzmann Distribution by Basic Computer Simulations. J. Chem. Educ., 97, 10, 3910-3913, https://dx.doi.org/10.1021/acs.jchemed.0c00559

Lemons D. S. (2013). A student's guide to entropy. Cambridge University Press, Cambridge, United Kingdom.

Magana A. J., \& Silva-Coutinho G. (2017). Modeling and simulation practices for a computational thinking-enabled engineering workforce. Comput. Appl. Eng. Educ. 25, 62-78.

Pantellini, F. G. E. (2000). A simple numerical model to simulate a gas in a constant gravitational field. American Journal of Physics 68, 61-68. http://doi.org/10.1119/1.19374

Reichl L. E. (2016). A Modern Course in Statistical Physics, $4^{\text {th }}$ edition. Wiley-VCH Verlag GmbH \& Co. KGaA, Weinheim, Germany.

Sarabando C., Cravino J. P., \& Soares A. A. (2014). Contribution of a computer simulation to students' learning of the physics concepts of weight and mass. Procedia Technol. 13, 112-21.

Schmidt-Böcking, H., Schmidt, L., Lüdde, H.J., Trageser W., Templeton A., \& Sauer T. (2016). The Stern-Gerlach experiment revisited. The European Physical Journal H 41, 327-364. https://doi.org/10.1140/epih/e2016-70053-2

Serway R. A., \& Jewett J. W. (2014). Physics for scientist and engineers with Modern Physics, $9^{\text {th }}$ edition. Brooks/Cole, Boston, United States.

Taub R., Armoni M., Bagno E., \& Ben-Ari M. M. (2015). The effect of computer science on physics learning in a computational science environment. Comput. Educ. 87, 10-23.

van Kampen, N. G. (2007). Stochastic processes in Physics and Chemistry, $3^{\text {td }}$ edition. North Holland, Amsterdam, the Netherlands.

Yetilmezsoy K., \& Mungan C. E. (2018). MATLAB time-based simulations of projectile motion, pendulum oscillation, and water discharge. European Journal of Physics, 39, $065803 \mathrm{https:// \textrm {doi } . 0 \mathrm { rg } / 1 0 . 1 0 8 8 / 1 3 6 1 - 6 4 0 4 / \text { aadaee }}$

Zupančič, B., \& Sodja, A. (2013). Computer-aided physical multi-domain modelling: Some experiences from education and industrial applications. Simul. Model. Pract. Th. 33, 45-67.

IJPCE - International Journal of Physics and Chemistry Education, 13(2), 33-45 


\section{Appendix}

Below you can find the codes that we have used for the simulations discussed in the actual paper. The crucial explanations are positioned as comments (underscored) in each script (Boriçi, 2019).

\section{maxwell1.m}

$\mathrm{n}=300 ; \mathrm{N}=50000 ;$ Sample combination

$\mathrm{Z}=2 *(\operatorname{rand}(\mathrm{n}, \mathrm{N})>0.5)-1$; $\frac{\circ}{\mathrm{O}}$ Generation uniformly distributed $1^{\prime} \mathrm{s}$ and $1^{\prime} \mathrm{S}$

$\overline{\mathrm{V} x \mathrm{x}}=\operatorname{sum}(\mathrm{Z}) ;$ 을 Velocity component generation

$r \max =5.5 *$ floor $(\operatorname{sqrt}(\mathrm{n}))$; $r$ step $=\max (1,2 *$ floor $(r \max / 100))$; $\frac{\circ}{\circ}$ Maximum value of speed, up to 5.5 times standard deviation. There should be a careful choice for large $\mathrm{n}$

$r=-r$ max:r_step:r_max; $\frac{\circ}{\circ}$ Interval along the $x$ axis

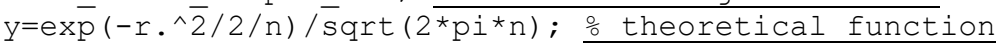

$\mathrm{h}=\mathrm{hist}(\mathrm{v} \mathrm{x}, \mathrm{r}) ;$ 응 Non-normalized empirical probabilites

$\mathrm{x}=\mathrm{r}(\mathrm{h} \sim=0) ; \mathrm{h}=\mathrm{h}(\mathrm{h} \sim=0) ;$ o The zero's are excluded

$\mathrm{p}=\mathrm{h} /(\operatorname{diff}(\mathrm{x}) * \mathrm{~h}(2$ :end)' $) ;$ o Normalized empirical probabilites

bar $(x, p) \div$ Plot of simulated data

hold on

plot $\left(r, y, r^{\prime}\right) \div$ Plot of theoretical function

\section{area_under.m}

$\mathrm{q}=\operatorname{cumsum}(\mathrm{p})$;

$\mathrm{k}=(\mathrm{x}<-\operatorname{sqrt}(\mathrm{n}))$; $\frac{\circ}{\circ}$ Area under curve for $\mathrm{x}<-\sigma$

$a=q(\operatorname{sum}(k)) / q($ end $)$

$l=(x>\operatorname{sqrt}(n))$; $\%$ Area under curve for $x>\sigma$

$\mathrm{b}=\mathrm{q}(\operatorname{sum}(\mathrm{l})) / \mathrm{q}($ end $)$

prob $=1-a-b \frac{\circ}{\circ}$ Area under curve for $-\sigma<\mathrm{x}<\sigma$

응 The operations are repeated in the same way for 20 and 30

\section{maxwell2.m}

$\mathrm{n}=300 ; \mathrm{N}=50000 ; \%$ Sample combination

$\mathrm{Z} 1=2 *(\operatorname{rand}(\mathrm{n}, \mathrm{N})>0.5)-1$; $\frac{\circ}{\mathrm{o}}$ Generation uniformly distributed $1^{\prime} \mathrm{s}$ and $\underline{\underline{1} \mathrm{~S}}$

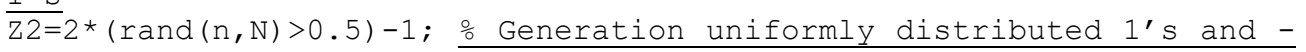

$\underline{1^{\prime} \mathrm{s}}$

$\overline{\mathrm{V} \mathrm{x}}=\operatorname{sum}(\mathrm{Z} 1) ; \mathrm{v} \quad \mathrm{y}=\operatorname{sum}(\mathrm{z} 2)$;

$\mathrm{v}=\operatorname{sqrt}\left(\mathrm{v}_{-} \mathrm{x} \cdot \wedge^{\wedge} 2+\mathrm{v}_{-} \mathrm{y} \cdot{ }^{\wedge} 2\right) ;$ o speed generation

$r_{\max }=5 . \overline{5} *$ floor $(\operatorname{sqrt}(\mathrm{n})) ; r_{-} \operatorname{step}=\max \left(1,2^{\star}\right.$ floor $\left.\left(r_{-} \max / 100\right)\right) ;$ \% Maximum value of speed, up to 5.5 times standard deviation. There should be a careful choice for large $\mathrm{n}$

$r=0: r$ step: $r$ max; $\frac{\circ}{\circ}$ Interval along the $\mathrm{x}$ axis for $i=1: r$ max +1

$y(i)=2{ }^{*} i^{\star} r(i) * \exp (-r(i) \wedge 2 / 2 / n) /\left(2 * p i{ }^{*}\right)$; $\frac{\circ}{\circ}$ theoretical function end

h=hist $(v, r)$; $\frac{\circ}{\circ}$ Non-normalized empirical probabilites $\mathrm{x}=\mathrm{r} ; \stackrel{\circ}{\circ}$ No need to exclude zero's

$\mathrm{p}=\mathrm{h} /\left(\overline{\mathrm{diff}}(\mathrm{x})^{\star} \mathrm{h}\left(2\right.\right.$ : end)'); $\frac{\circ}{\circ}$ Normalized empirical probabilites bar $(x, p) \div$ Plot of simulated data

hold on

plot $(r, y, ' r ') \div$ Plot of theoretical function

\section{maxwell3.m}

$\mathrm{n}=300 ; \mathrm{N}=50000 ;$ Sample combination

$\mathrm{Z} 1=2 *(\operatorname{rand}(\mathrm{n}, \mathrm{N})>0.5)-1 ;$ 응 Generation uniformly distributed $1^{\prime} \mathrm{s}$ and $\underline{1 / \mathrm{s}}$

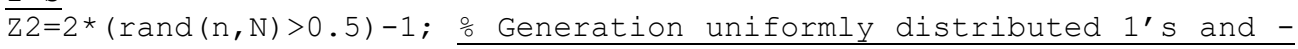
$\frac{1^{\prime} \mathrm{s}}{\mathrm{Z} 3=2}$

$1^{\prime} \mathrm{s}$

$\overline{\mathrm{V} X}=\operatorname{sum}(\mathrm{Z} 1) ; \mathrm{V} \quad \mathrm{y}=\operatorname{sum}(\mathrm{Z} 2) ; \mathrm{V} \quad \mathrm{z}=\operatorname{sum}(\mathrm{z} 3)$.

$\mathrm{v}=\operatorname{sqrt}\left(\mathrm{v} x \cdot{ }^{\wedge} 2+\mathrm{v} \mathrm{y} \cdot \cdot^{\wedge} 2+\mathrm{v} z \cdot{ }^{\wedge} 2\right) ; \frac{\mathrm{O}}{\circ}$ Speed generation

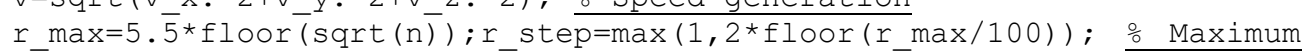
value of speed, up to 5.5 times standard deviation. There should be a careful choice for large $\mathrm{n}$

$r=0: r$ step: $r$ max; $\frac{\circ}{0}$ Interval along the $x$ axis

for $i=1: r \max +1$

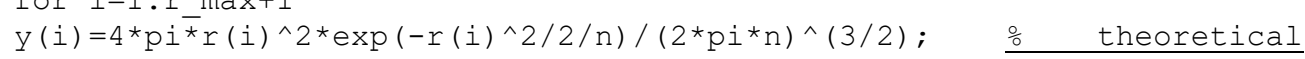
function

end

$\mathrm{h}=\mathrm{hist}(\mathrm{v}, \mathrm{r})$; $\frac{\circ}{\circ}$ Non-normalized empirical probabilites $\mathrm{x}=\mathrm{r} ;$ 을 no need to exclude zero's

$\mathrm{p}=\mathrm{h} /\left(\operatorname{diff}(\mathrm{x}) * \mathrm{~h}\left(2\right.\right.$ :end)'); $\frac{\circ}{\circ}$ Normalized empirical probabilites bar $(x, p) \div$ Plot of simulated data

hold on

plot $\left(r, y, r^{\prime}\right) \div$ Plot of theoretical function 